\title{
Eps8 regulates cellular proliferation and migration of breast cancer
}

\author{
CHENG CHEN $^{1 *}$, ZHONGHENG LIANG $^{1 *}$, WENHUAN HUANG ${ }^{1}$, XINXIN LI $^{1}$, FANGLIANG ZHOU $^{2}$, \\ XIANG HU $^{1}$, MEI HAN ${ }^{1}$, XIAOFENG DING ${ }^{1}$ and SHUANGLIN XIANG ${ }^{1}$ \\ ${ }^{1}$ Key Laboratory of Protein Chemistry and Development Biology of State Education Ministry of China, \\ College of Life Science, Hunan Normal University; ${ }^{2}$ College of Basic Medical Sciences, \\ Hunan University of Chinese Medicine, Changsha, P.R. China
}

Received August 19, 2014; Accepted October 2, 2014

DOI: 10.3892/ijo.2014.2710

\begin{abstract}
The role of Eps8 in human breast cancer was studied, and we found that Eps8 was overexpressed in $>60 \%$ of human breast cancer samples compared with adjacent normal breast tissues by immunohistochemical analysis. Eps8 was highly expressed in the highly invasive breast cancer cell line MDA-MB-231 compared with the weakly invasive breast cancer cell lines MCF7 and MDA-MB-468. MCF7 cell line stably expressing Eps8 was established by G418 screening, and the ectopic expression of Eps8 enhanced MCF7 breast cancer cell growth and survival as assessed by MTT analysis, cell viability and liquid colony formation, whereas the lentiviral expression of Eps8 shRNA in MDA-MB-231 cells resulted in a significant reduction in cellular growth and proliferation in vitro and in vivo. Furthermore, Eps8 knockdown inhibited breast cancer cell migration in wound healing assays, decreased the number and size of EGF-induced filopodia and increased the sensitivity of breast cancer cells to cisplatin analyzed by MTT assays. Eps8 knockdown decreased the levels of phosphorylated extracellular signal-regulated protein kinase (ERK) and MMP9 but increased p53. Moreover, Eps8 knockdown suppressed a partial EMT-like transition and showed a significant increase in E-cadherin and decrease in $\mathrm{N}$-cadherin and vimentin. These results suggest that Eps8 is overexpressed in human breast cancers, possibly by regulating
\end{abstract}

Correspondence to: Dr Xiaofeng Ding or Dr Shuanglin Xiang, Key Laboratory of Protein Chemistry and Development Biology of State Education Ministry of China, College of Life Science, Hunan Normal University, Changsha 410081, Hunan, P.R. China

E-mail: fengxiaoding@hotmail.com

E-mail: xshlin@hunnu.edu.cn

${ }^{*}$ Contributed equally

Key words: EGF receptor substrate 8, breast cancer, cellular growth and migration, lentiviral RNAi system, extracellular signal-regulated protein kinase, p53, F-actin, cisplatin, epithelial-to-mesenchymal transition, E-cadherin, $\mathrm{N}$-cadherin, vimentin
ERK signaling, MMP9, p53 and EMT-like transition to affect breast cancer cell growth, migration and invasion. Therefore, Eps8 might represent a novel potential target in human breast cancer therapy.

\section{Introduction}

Breast cancer is the most commonly malignancy of women worldwide (1), the primary cause of the development and progression of breast cancer is multiple genetic changes, whose accumulation results in the metastatic invasion (2). Some critical genes including HER-2/neu, p53, nm23, and cyclin $\mathrm{D}$ are studied in clinical diagnosis for prognostic or predictive outcome (3-6). Therefore, further understanding the molecular etiology of breast cancer is helpful for estimating disease prognosis and guiding treatment.

Eps8 extensively functions as an oncogene in various types of human solid tumors, including squamous cell carcinoma, pancreatic cancer and cervical cancer, as well as colon cancer, pituitary tumors and glioma (7-13) and even hematologic malignancies (14). High expression and concomitant tyrosine phosphorylation of Eps8 were detected in a serial of human tumor cell lines (15). Moreover, elevated Eps8 in cancer patients might be a biomarker of poor prognosis for decreased overall survival $(8,14,16)$. Eps 8 could increase cell growth and motility, by modulating the downstream pathways including the mTOR/STAT3/FAK, PI3K/AKT/ FOXM1/MMP9, p53/p21WAF1/CIP1-dependent pathway, EGFR signaling via Rac, and Ras/Raf/MEK/ERK pathways in cancer cells, thus affecting EGFR endocytosis, actin dynamics, RNA processing, cell cycle progression, angiogenesis, cell migration and invasion $(7-9,11,12)$. Furthermore, the effects of downregulated expression of Eps8 on chemotherapeutic agents are also studied. Mithramycin downregulates Eps8 expression and inhibits human epithelial carcinoma cell proliferation and migration (17). Additionally, the cytotoxicity of cisplatin is increased in Eps8-attenuated cervical cancer and lung cancer cells $(8,18)$. Moreover, the loss of Eps 8 protein in colorectal adenoma and carcinoma plays a role in the development of a subset of colorectal cancers, suggesting the significance of personalized medicine in patient treatment (19). 
Although Eps8 plays a critical role in the development of many malignancies (20), the expression and function of Eps8 in breast cancer are not clear. Combined cDNA array comparative genomic hybridization (CGH) and serial analysis of gene expression analysis (SAGE) identified candidate amplicon target Eps8 as novel putative oncogene in breast cancer (21). In this study, we further determine whether Eps8 is involved in breast cancer malignancy, including cell proliferation and migration. Eps8 was found overexpressed in breast cancer samples and highly invasive cell lines. Overexpression of Eps8 promoted MCF7 cell growth, whereas downregulation of Eps8 in MDA-MB-231 cells significantly inhibited cell proliferation, migration and cell motility. These data suggest that Eps8 serves as a novel growth regulator in breast cancer cells and could be a target for the diagnosis and treatment of breast cancer.

\section{Materials and methods}

Immunohistochemistry. Seventy-two breast cancer samples were examined and 3 adjacent normal mammary tissues were used as the control. The study was approved by the Hunan Normal University Human Ethics Committee, and informed consent was obtained from all patients. The immunohistochemical analysis was performed on polyformalin-fixed and paraffin-embedded tissues. Sections $(5 \mu \mathrm{m})$ were deparaffinized by two 10 -min washes in xylene, then rehydrated through successive graded ethanol solutions. Endogenous peroxidase was quenched with $3 \% \mathrm{H}_{2} \mathrm{O}_{2}$ in methanol for $10 \mathrm{~min}$ and washed for $5 \mathrm{~min}$ in PBS. Antigen retrieval was achieved by microwaving sections in $0.01 \mathrm{M}$ citrate buffer ( $\mathrm{pH}$ 6.0) for $10 \mathrm{~min}$ at $800 \mathrm{~W}$. The tissues were blocked in $5 \%$ bovine serum albumin (BSA) in PBS for $1 \mathrm{~h}$ before the addition of the mouse monoclonal anti-Eps8 antibody or mouse IgG control (diluted 1:500, BD Biosciences, CA, USA) at $4^{\circ} \mathrm{C}$ for overnight. The sections were incubated with HRP-conjugated goat anti-mouse secondary antibody (diluted 1:100, Sigma-Aldrich, St. Louis, MO, USA) for $45 \mathrm{~min}$ and then with 3,3-diaminobenzidine (DAB) $/ \mathrm{H}_{2} \mathrm{O}_{2}$ for $10 \mathrm{~min}$. Sections were counterstained with hematoxylin, mounted and photographed using an optical microscope (Olympus CX41, Tokyo, Japan). The percentage of tumor cells stained was scored as: 0 (no cell staining), + or $1(\leq 30 \%),++$ or $2(31-60 \%)$ and +++ or $3(61-100 \%)$. The staining between two score values was given 0.5 .

Cell culture. Human breast cancer cells MDA-MB-231, MCF7 and T47D were cultured in RPMI-1640 medium (Gibco BRL, Grand Island, NY, USA) containing $100 \mathrm{U} / \mathrm{ml}$ penicillin, $100 \mu \mathrm{g} / \mathrm{ml}$ streptomycin and $10 \%$ fetal bovine serum (FBS, Hyclone, Australia). MDA-MB-453, MDA-MB-468 cells and normal mammary cells HBL100 were cultured in Dulbecco's modified Eagle's medium (DMEM, Gibco) supplemented as described above. All cells were kept at $37^{\circ} \mathrm{C}$ in a humidified atmosphere with $5 \% \mathrm{CO}_{2}$.

Establishment of MCF7 cell lines overexpressing GFP and GFP-Eps8. MCF7 cells were transiently transfected with constructed pEGFP-C3-Eps8 or pEGFP-C3 control plasmids using Lipofectamine 2000 (Invitrogen, Carlsbad, CA, USA) (13). Stably transfected cells were screened by $400 \mu \mathrm{g} / \mathrm{ml} \mathrm{G} 418$ (Sigma) $(22,23)$. The transfection efficiency was detected using an invert fluorescence microscope (Olympus IX71, Tokyo, Japan).

Eps8 RNAI lentivirus generation. Efficient siRNA sequence targeting Eps8 (NM_004447.5) was from the position 93-111 relative to the start codon, stem-loop DNA oligonucleotides were synthesized by Shanghai GeneChem Co. Ltd., China and inserted into the lentivirus-based RNAi vector pGCSIL-GFP (GeneChem) (13). A non-targeting stem-loop DNA was also cloned into pGCSIL-GFP vector as a negative control (NC). Lentiviral particles were prepared as described previously (24). Briefly, the lentivirus expression plasmid and packaging plasmids (pHelper 1.0 and pHelper 2.0) were cotransfected into $293 \mathrm{~T}$ cells, supernatants were harvested $48 \mathrm{~h}$ after transfection and filtered through a $0.45-\mu \mathrm{m}$ pore size filter (Millipore, Billerica, MA, USA) and concentrated by ultracentrifugation. The infectious titer was determined using hole-by-dilution titer assay. MDA-MB-231 cells at a density of 100,000 cells per well in 6-well plates were infected with Eps8-RNAi-lentivirus or NC-RNAi-lentivirus and $5 \mu \mathrm{g} / \mathrm{ml}$ polybrene (Sigma) at the multiplicity of infection (MOI) 10 and detected on the 4th day by the invert fluorescence microscope.

Cell proliferation assays. For cell viability assay, 3,000 cells were seeded in octuplicate in 96-well plates untreated or treated with $30 \mu \mathrm{M}$ of cisplatin (DDP, Sigma) for $24 \mathrm{~h}$. On days 1,3 and 4 or days 1,3 and 6 , cells were analyzed with $1 \mathrm{mg} / \mathrm{ml}$ 3-(4,5-dimethylthiazol-2-yl)-2,5-diphenytetra zolium bromide (MTT, Sigma) at $37^{\circ} \mathrm{C}$ for $4^{\circ} \mathrm{h}$. Then $100 \mu \mathrm{l}$ dimethylsulfoxide (DMSO)/well was added to dissolve the formazan crystals. The absorbance at $490 \mathrm{~nm}$ was obtained using a spectrophotometer (UV-2102C, Changsha, China).

For cell survival assay, 100,000 MCF7 cells stably expressing GFP-Eps8 or GFP and parental cells were plated in triplicate in 6-well plates in complete medium containing $400 \mu \mathrm{g} / \mathrm{ml} \mathrm{G} 418$. MDA-MB-231 cells $(50,000)$ infecting Eps8-RNAi-LV or NC-RNAi-LV were plated in triplicate in 6-well plates. After 3-6 days, viable cell numbers were counted with a hemocytometer after trypan blue staining of dead cells.

Liquid colony formation was performed, 2,000 cells were seeded in triplicate in 6-well plates and grown in complete culture medium for $>10$ days. Colonies were fixed with methanol, stained with Giemsa (BBI International, Cardiff, UK) and photographed with a digital camera (Canon IXUS $125 \mathrm{HS}$, Tokyo, Japan). Only colonies containing $>30$ cells were counted under an inverted microscope (Zeiss Axiovert 25, LLC, USA). All experiments were carried out at least three times.

Cell cycle analysis. Lentivirus-infected and parental MDA-MB-231 cells were plated onto 6-well plates for $24 \mathrm{~h}$. Serum was withdrawn when cells were $70 \%$ confluent. After $36 \mathrm{~h}, 10 \%$ FBS was added in the medium for an additional $18 \mathrm{~h}$. Cells were collected gently, fixed in 70\% ethanol and stained with propidium iodide (PI, BD Pharmingen, San Diego, CA, USA), the DNA content was analyzed on a BD FACSCalibur cytometer (Becton-Dickinson, San Jose, CA, USA) using CellQuest Pro and ModFit software (BD).

Apoptosis assay.Lentivirus-infected and parental MDA-MB-231 cells were harvested and washed with cold PBS. The pellet 
was resuspended in $1 \mathrm{X}$ binding buffer and the sample solution was incubated with PI and FITC-conjugated Annexin V (BD Pharmingen) for $15 \mathrm{~min}$ at room temperature in the dark. The samples were analyzed by the BD FACSCalibur cytometer using the CellQuest software.

Tumor formation in nude mice. The mouse experiments were carried out according to the ethical guidelines for laboratory animal use and approved by the Ethics Committee of Hunan Normal University. Approximately $10^{7}$ of lentivirus-infected MDA-MB-231 cells in $0.2 \mathrm{ml}$ of sterile PBS were injected subcutaneously into the left and right dorsal regions of 4- to 5 -week-old female nude mice (BALb/c). Mice were checked every 2 days and the formed tumors were measured with a micrometer (25). After 26 days, mice were sacrificed, and tumors were excised, weighed and photographed.

Wound-healing migration assays. MDA-MB-231 cells were cultured in 24 -well plates until $>90 \%$ confluence. A $100-\mu 1$ pipette tip was used to generate wounds. After wound creation, the medium was changed to remove cellular debris. Three wounded areas in each well were marked on the bottom of plates and photographed at 1 and 4 days with an invert microscope.

Immunofluorescence assays. Stimulation of membrane ruffling with $60 \mathrm{ng} / \mathrm{ml}$ of human EGF (Sigma, Deisenhofen, Germany) in RPMI-1640 for 30 min was performed after serum deprivation for 48 h. MDA-MB-231 cells were fixed with $3.7 \%$ paraformaldehyde for $10 \mathrm{~min}$, permeabilized with $0.1 \%$ Triton $\mathrm{X}-100$ for 10 min, and blocked with 5\% BSA in PBS. Alexa fluor 595 conjugated to phalloidin (diluted 1:500, Molecular Probes, Eugene, OR, USA) and $1 \mu \mathrm{g} / \mathrm{ml}$ Hoechst 33258 (Sigma) were incubated for 20 min to stain F-actin and the nucleus in the dark. All specimens were viewed with an upright fluorescence microscope (Zeiss Axioskop 2, LLC, USA). At least 20 cells were measured in three independent experiments.

Western blot analysis. Cells were lysed in RIPA buffer [50 mM Tris- $\mathrm{HCl}$ (pH 7.2), $150 \mathrm{mM} \mathrm{NaCl}, 1 \%$ Triton $\mathrm{X}-100,1 \%$ sodium deoxycholate, $0.1 \%$ SDS and cocktail protease inhibitors]. The lysates $(50 \mu \mathrm{g})$ were denatured in sample buffer and heated to $105^{\circ} \mathrm{C}$ for $5 \mathrm{~min}$. Samples were then separated on $10-15 \%$ SDS-PAGE gels and transferred to polyvinylidene difluoride (PVDF) membranes (Millipore). The membranes were blocked with $1 \%$ BSA in TBS-T [10 mM Tris- $\mathrm{HCl}$ (pH 7.5), $150 \mathrm{mM} \mathrm{NaCl}, 0.1 \%$ Tween-20] and incubated overnight with rabbit polyclonal antibodies against GFP and MMP9, mouse monoclonal antibodies against ERK and phosphorylated ERK, cyclin D1 (CCND1), c-Myc, p53, N-cadherin, E-cadherin, vimentin, $\beta$-actin and GAPDH (diluted 1:1,000, Santa Cruz Biotechnology, Santa Cruz, CA, USA) in TBS-T containing $1 \%$ BSA with gentle shaking at $4^{\circ} \mathrm{C}$. HRP-conjugated goat anti-rabbit and goat anti-mouse secondary antibodies (diluted 1:5,000, Sigma) were used. The signals were detected with SuperSignal West Pico chemiluminescent Substrate (Thermo Scientific Pierce, Rockford, IL, USA) and visualized with tanon-5200 system (Bio-tanon, Shanghai, China).

Statistical analysis. All statistical analyses were performed using the SPSS 11.0 software (SPSS Inc., Chicago, IL, USA).
Table I. Patient characteristics of immunohistochemistry.

\begin{tabular}{|c|c|}
\hline Variable & No. of patients $(\%)$ \\
\hline Total number & $72(100)$ \\
\hline \multicolumn{2}{|c|}{ Age (median, 48 years) } \\
\hline$<48$ years & $33(45.8)$ \\
\hline$\geq 48$ years & $39(54.2)$ \\
\hline \multicolumn{2}{|c|}{$\begin{array}{l}\text { Histological diagnosis } \\
\text { (invasive carcinoma) }\end{array}$} \\
\hline Ductal & $61(84.7)$ \\
\hline Medullary & $11(15.3)$ \\
\hline \multicolumn{2}{|c|}{$\begin{array}{l}\text { Histological grade } \\
\text { (invasive carcinoma) }\end{array}$} \\
\hline Grade 1 & $14(19.4)$ \\
\hline Grade 2 & $40(55.6)$ \\
\hline Grade 3 & $18(25)$ \\
\hline \multicolumn{2}{|l|}{ TNM staging } \\
\hline Stage IV & $4(5.6)$ \\
\hline Stage III & $22(30.6)$ \\
\hline Stage II & $39(54.2)$ \\
\hline Stage I & $7 \quad(9.6)$ \\
\hline Normal tissue & 3 \\
\hline
\end{tabular}

Data are shown as mean \pm SD from at least 3 independent experiments. Statistical significance was determined using Student's t-test at P-values $<0.05$.

\section{Results}

Eps 8 was overexpressed in breast cancer tissues and highinvasive cell lines. The expression level of Eps8 was examined in 4 stage IV, 22 stage III, 39 stage II, 7 stage I human breast cancers and 3 adjacent normal mammary tissues by the immunohistochemistry analysis using mouse monoclonal anti-Eps8 antibody. We found that Eps8 was completely localized in the cytoplasm (Fig. 1A). Eps8 expression was detected in $46(63.9 \%)$ of the 72 breast cancers with strong staining (3+), $21(29 \%)$ of the 72 breast cancers were moderately stained $(2+)$, and $5(6.9 \%)$ were weakly stained or negative for Eps8 expression (+/0), which indicated Eps8 was highly expressed in breast cancers $(\mathrm{P}<0.001)$ according to Student's t-test. A complete loss of Eps8 expression was observed in normal mammary tissues (Fig. 1B). Therefore, Eps8 expression was significantly increased in human breast cancer samples. Patient characteristics are summarized in Table I.

We next analyzed the expression of Eps8 proteins in five human breast cancer cell lines. Higher expression of Eps8 proteins was evident in MDA-MB-231, MDA-MB-453 and T47D cells than in MDA-MB-468, MCF7 cells and normal breast epithelial cells HBL100 (Fig. 1C). Thus, the level of Eps8 expression was high in the highly invasive breast cancer cell line MDA-MB-231 and low in the weakly invasive breast 

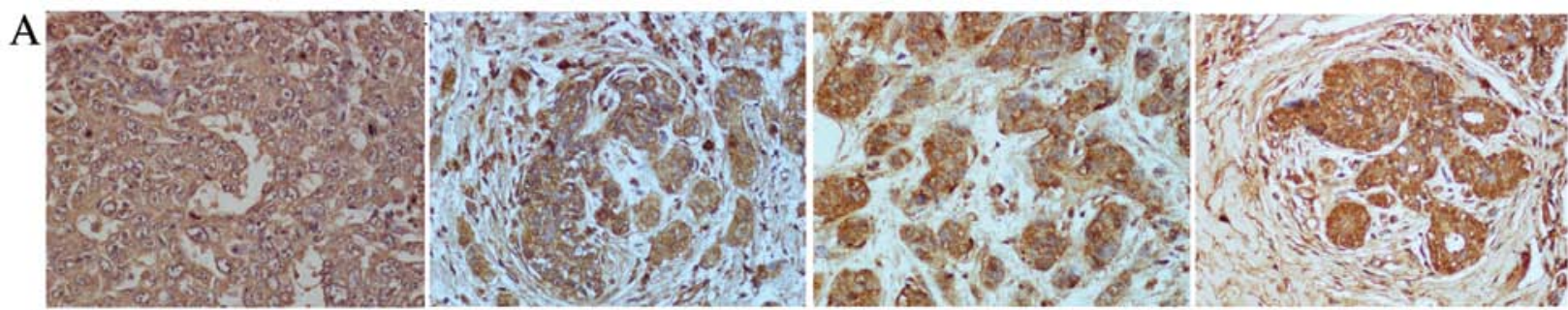

Invasive ductal carcinoma

Invasive ductal carcinoma Invasive ductal carcinoma

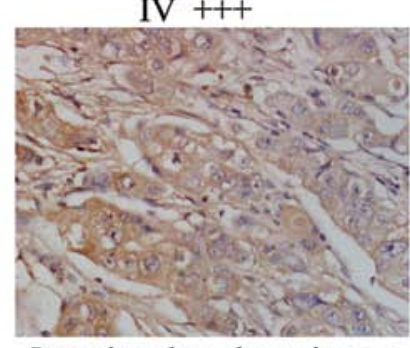

Invasive ductal carcinoma

$\mathrm{I}++$

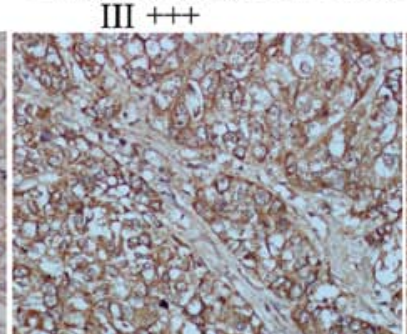

Medullary carcinoma

III +++
$\mathrm{II}+++$

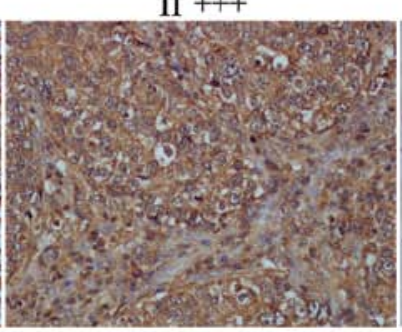

Medullary carcinoma

II +++

\section{Invasive ductal carcinoma}

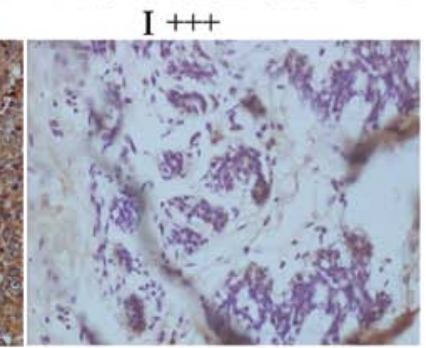

Adjacent normal tissue

0
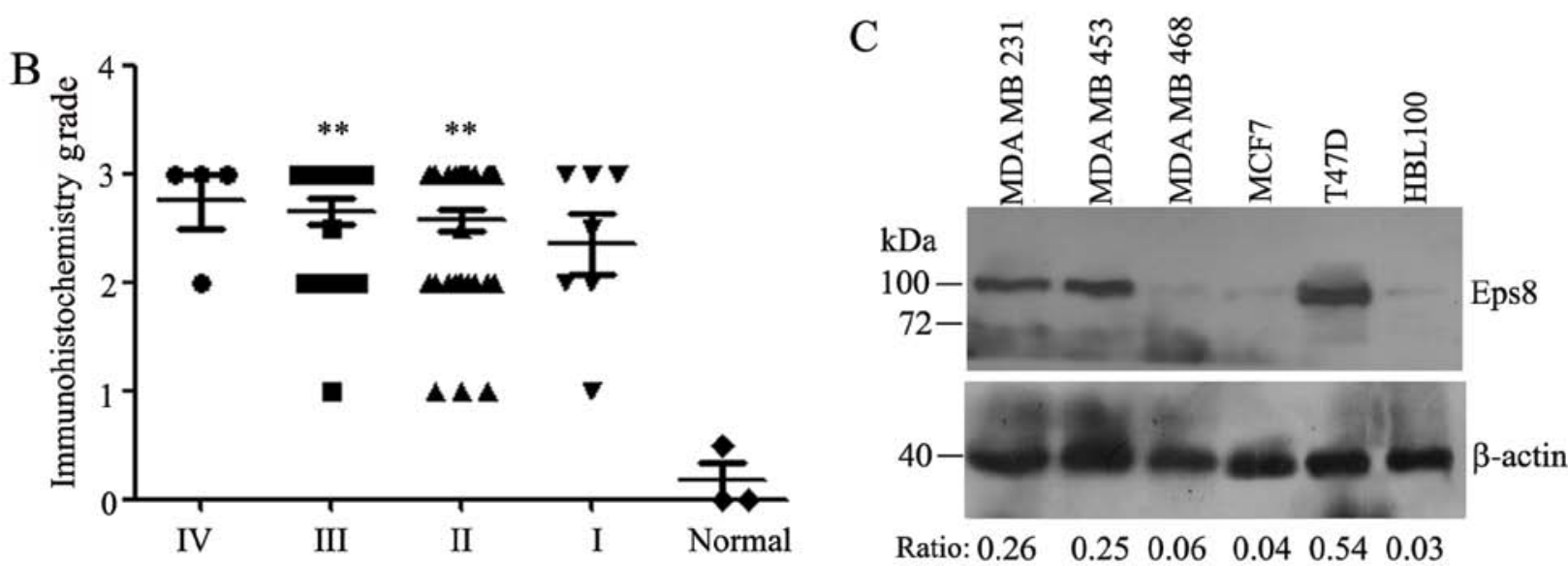

Ratio: $\begin{array}{llllll}0.26 & 0.25 & 0.06 & 0.04 & 0.54 & 0.03\end{array}$

Figure 1. The expression levels of Eps8 in breast cancer tissues and cell lines. (A) Representative IHC staining of Eps8 in 72 mammary tumors and 3 adjacent normal samples as examined by immunohistochemical analysis. Strong cytoplasmic expression of Eps8 (brown staining) was detected from stages I-IV breast cancer tissues while the nucleus was stained blue with hematoxylin. Sections were viewed at $\mathrm{x} 400$ magnification. (B) Immunohistochemical grades of breast cancers and normal mammary tissues stained with monoclonal anti-Eps8 antibody. The staining intensity was scored with grades 0-3. The data were analyzed by GraphPad Prism 5 software. Each symbol represents an individual sample. Statistical comparisons between breast cancers and normal tissues were performed according to Student's t-test. ${ }^{* *} \mathrm{p}<0.001$. (C) Eps8 protein levels in different breast cancer cell lines as detected by western blotting. $\beta$-actin was used as an internal control. Images were quantified by Gel-Pro Analyzer 4.0 software.

cancer cell line MCF7. Both cell lines were further selected to knockdown and overexpress Eps8 proteins, respectively.

Eps8 overexpression enhances the proliferation of MCF 7 cells. To further investigate the role of Eps8 in human breast cancer cells, we overexpressed Eps8 and asked whether Eps8 upregulation enhances the proliferation of breast cancer cells The pEGFP-C3/Eps8 and pEGFP-C3 plasmids were transfected to established MCF7 cell lines stably overexpressing GFP/Eps8 and GFP by G418 screening (Fig. 2A). As shown in Fig. 2B, GFP and GFP/Eps8 target proteins were expressed at high levels in MCF7 cells.

We then investigated whether Eps8 overexpression in the breast cancer MCF7 cells leads to cell proliferation. The same amount of cells was plated in 96-well plates, and we examined cell viability using MTT assays, Eps8 overexpression resulted in a remarkable increase in viable cells (Fig. 2C). The same amount of cells was plated in triplicate in 6-well plates and cell number was counted on days 1, 3 and 4 . We found that the overexpression of Eps8 in MCF7 cells shows an increased cellular growth compared with controls (Fig. 2D). Further, the liquid colony formation assays indicated a great increase in colony number and size (Fig. 2E). Therefore, these results suggested that Eps8 could contribute to breast cancer cell survival and proliferation in vitro.

Eps8 knockdown inhibits the proliferation and survival of MDA-MB-231 cells in vitro and in vivo. The above results indicated that Eps 8 overexpression significantly promotes MDA-MB-231 cellular proliferation. To gain further supporting data, we attempted to knock down Eps8 expression and asked whether shRNA-mediated Eps8 knockdown inhibits the proliferation of breast cancer cells. Then, lentivirusbased RNAi vector pGCSIL-Eps8 containing the efficient 


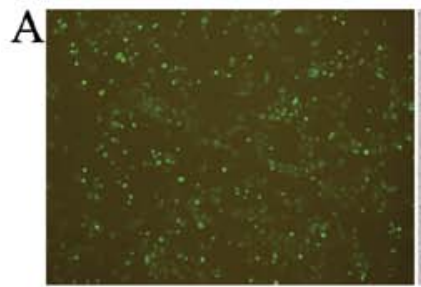

GFP (fluorescent)

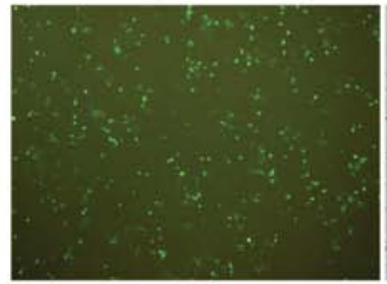

GFP-Eps8 (fluorescent)

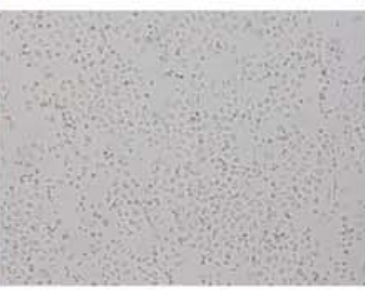

GFP (grey)

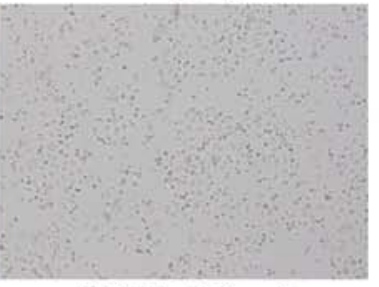

GFP-Eps8 (grey)
B

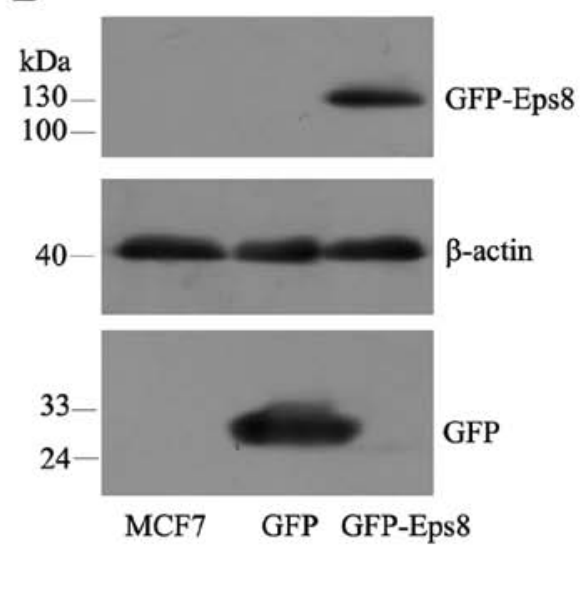

$\mathrm{C}$
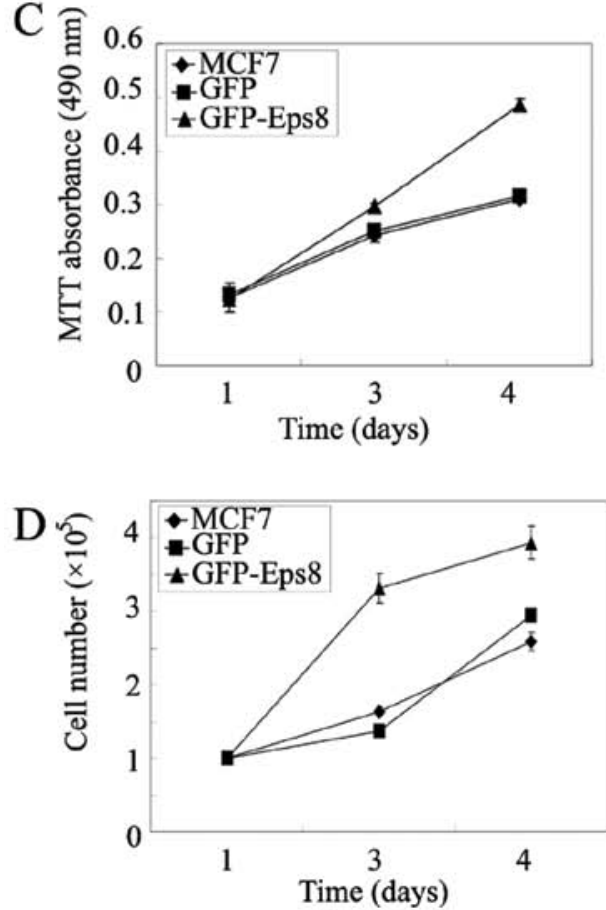

$\mathrm{E}$
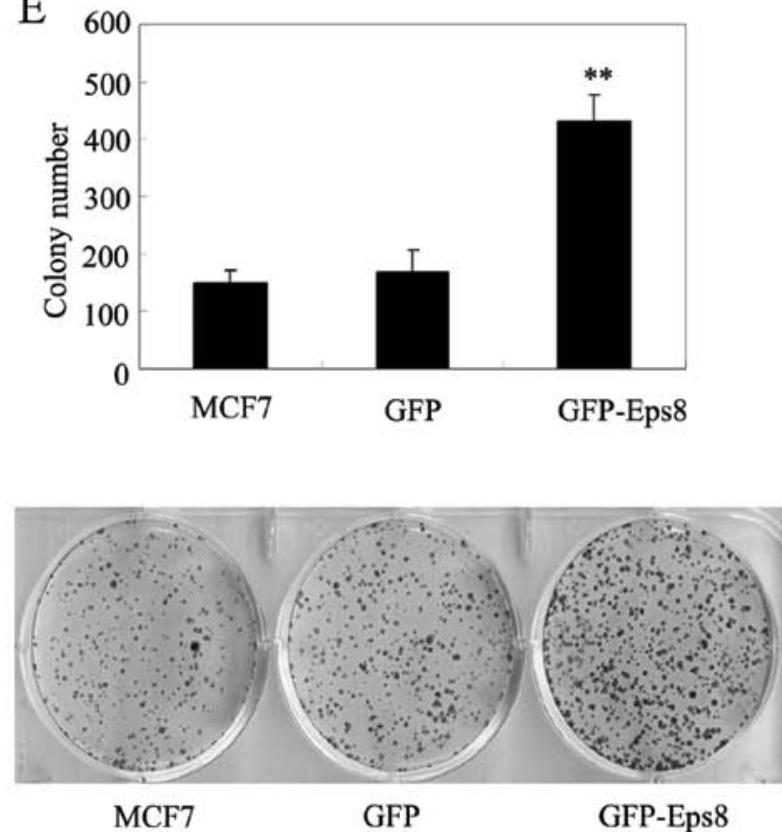

Figure 2. Effects of Eps8 overexpression on MCF7 breast cancer cell proliferation. (A) Stable overexpression of GFP-Eps8 and GFP in MCF7 cells by G418 screening was examined by immunofluorescence assay. (B) GFP and GFP/Eps8 expression in MCF7 cells was confirmed by western blotting using anti-GFP antibodies. $\beta$-actin served as a loading control. (C) MTT assays in mock or transfected MCF7 cells. Cells (3,000) were plated in octuplicate in 96-well plates and grown in RPMI-1640 with 10\% FBS. The absorbance was analyzed for 1, 3 and 4 days. (D) Cell survival assay in GFP-Eps8-transfected MCF7 cells as compared with GFP-transfected and parental MCF7 cells. Cells $(100,000)$ were plated into 6-well plates in triplicate, grown in RPMI-1640 with $10 \%$ FBS for 1, 3 and 4 days and stained with trypan blue in PBS, viable cells were counted. (E) Representative liquid colony formation analysis in mock or transfected MCF7 cells. Cells $(2,000)$ were seeded in triplicate in 6-well plates, and grown for 13 days. Colonies were fixed with methanol, stained with Giemsa, counted (upper panel) and images were taken (lower panel). These data represent at least 3 independent experiments with similar results. ${ }^{* *}$ p $<0.01$, compared with parental and control cells.

Eps8 shRNA1 and packaging plasmids were cotransfected to $293 \mathrm{~T}$ cells (13). Lentiviral particles were prepared to infect MDA-MB-231 cells. The fluorescence intensity was markedly increased 4 days after infection and the infection efficiency was close to $90 \%$ in MDA-MB-231 cells (Fig. 3A). Additionally, western blot analysis showed that Eps8 shRNA1 could significantly suppress the expression of endogenous Eps8 proteins when compared with negative control shRNA (Fig. 6A).

Next, we examined whether Eps8 is a critical regulator of breast cancer cell proliferation and investigated the effect of Eps8 knockdown on MDA-MB-231 breast cancer cell growth. From Fig. 3B and C, we observed that Eps8 deletion significantly inhibits the proliferation of MDA-MB-231 cells, whereas control shRNA has no effect. The liquid colony formation assays showed that Eps8 knockdown displays much fewer and smaller colonies in MDA-MB-231 cells, while control shRNA has no effect compared with uninfected parental cells (Fig. 3D). We further explored the molecular mechanism involved in Eps8-mediated cancer cell survival, the effects of Eps8 knockdown on cell cycle and apoptosis were investigated. The flow cytometry analysis showed that 


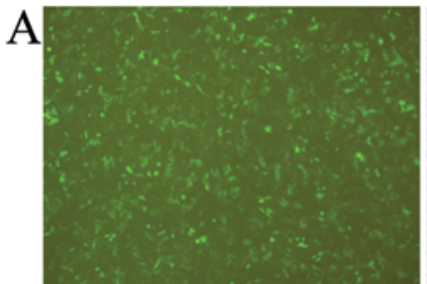

Eps8-shRNA-LV (fluorescent)

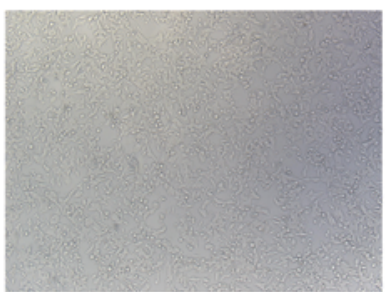

Eps8-shRNA-LV (grey)

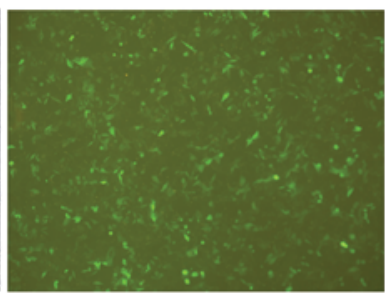

NC-shRNA-LV (fluorescent)

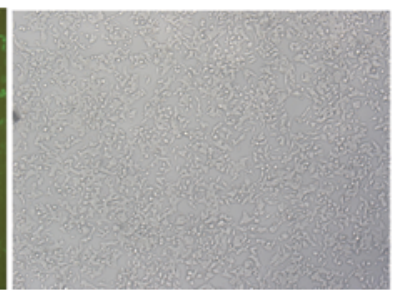

NC-shRNA-LV (grey)
$\mathrm{B}$

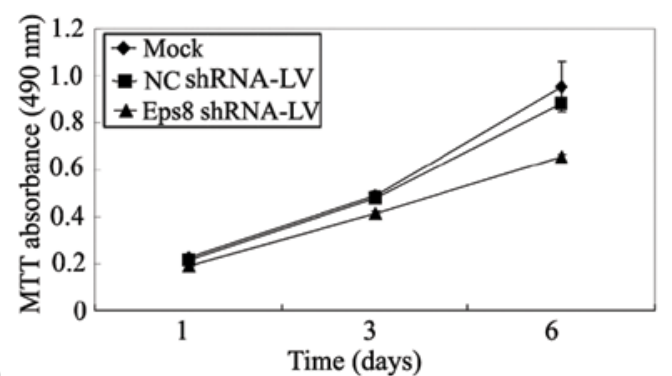

$\mathrm{C}$

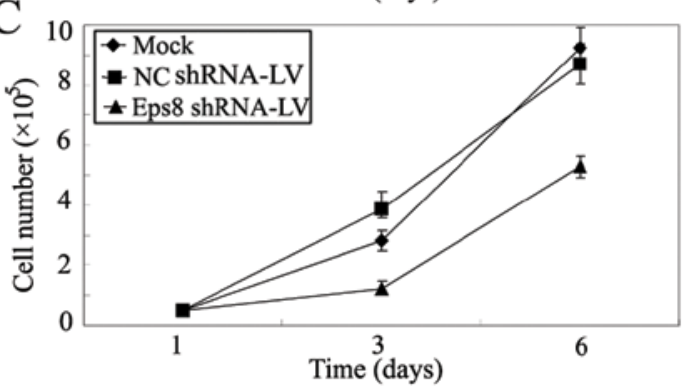

D
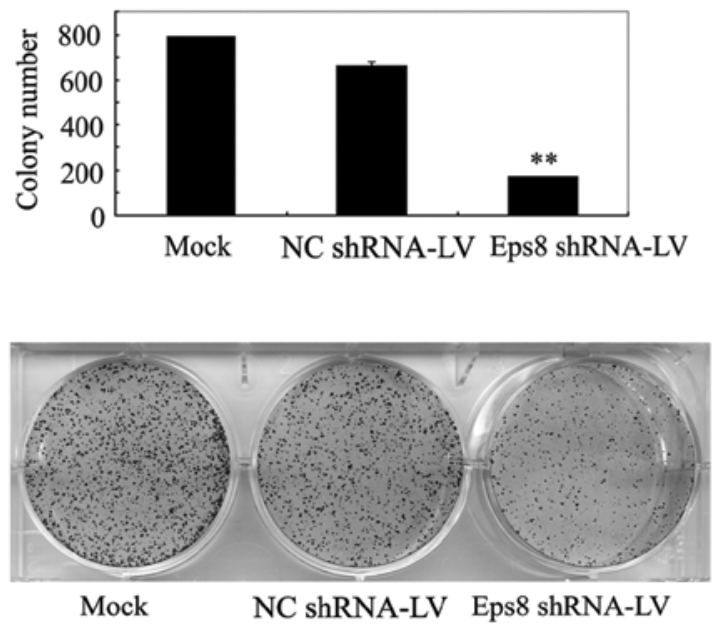

\section{E}

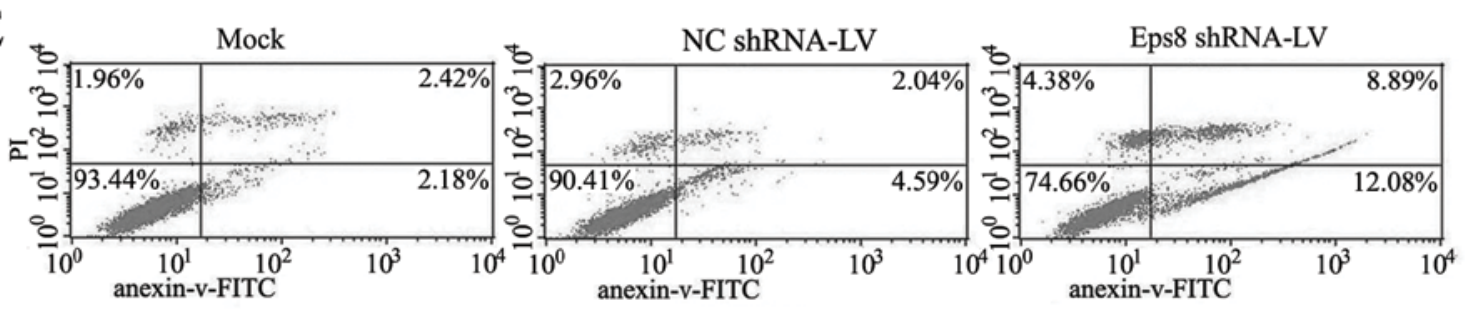

$\mathrm{F}$

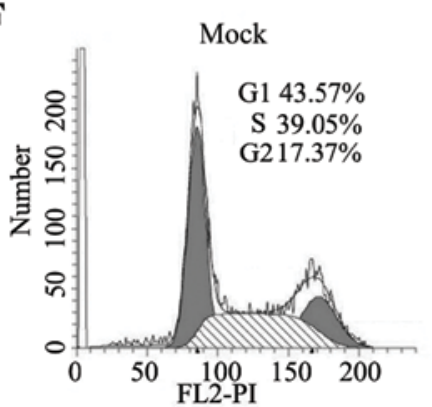

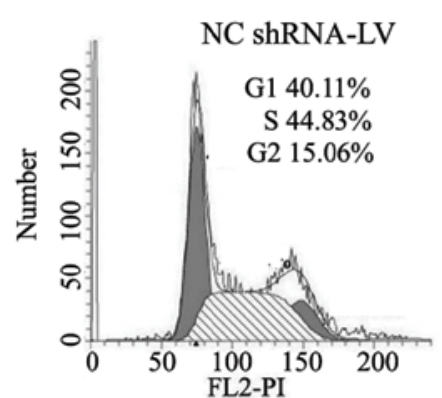

Figure 3. Effects of Eps8 knockdown on the proliferation and cell cycle progression of breast cancer cells MDA-MB-231. (A) Representative images of immunofluorescence assays for the measurement of the transduction efficiency of MDA-MB-231 cells infected with Eps8 shRNA1 LV and NC shRNA LV. (B) MTT assays in mock or infected MDA-MB-231 cells. Cells (3,000) were plated and grown. The absorbance was analyzed for 1, 3 and 6 days. (C) Cell survival assay in Eps8 shRNA1-infected MDA-MB-231 cells as compared with NC shRNA-infected and parental MDA-MB-231 cells on 1st, 3rd and 6th days. (D) Representative liquid colony formation analysis in mock or infected MDA-MB-231 cells. Cells $(2,000)$ were seeded and grown for 2 weeks. Colonies were fixed with methanol, stained with Giemsa, counted (upper panel) and taken images (lower panel). (E) Comparison of DNA contents between lentivirus-infected and parental MDA-MB-231 cells by flow cytometry. The DNA content of infected cells starved by withdrawal of serum for $36 \mathrm{~h}$ followed by the addition of serum for $18 \mathrm{~h}$ was evaluated. (F) Lentivirus-infected and parental MDA-MB-231 cells were scored for apoptosis by FACS analysis. Cells were stained with Annexin V and PI, followed by flow cytometric analysis. The results are expressed as mean \pm SD of 3 independent experiments. ${ }^{* *}<<0.01$.

Eps8 knockdown results in a significant decrease from mean 91.9 to $74.66 \%$ in living MDA-MB-231 cells and exhibits an increase in early and late apoptotic cells compared to control shRNA-infected and parental cells (Fig. 3E). Moreover, we compared the DNA content between Eps8 repressed and control cells and found that the proportion of S-phase cells was significantly decreased in Eps8 knockdown cells (31.33\%) compared with MDA-MB-231 cells (39.05\%), and the propor- 


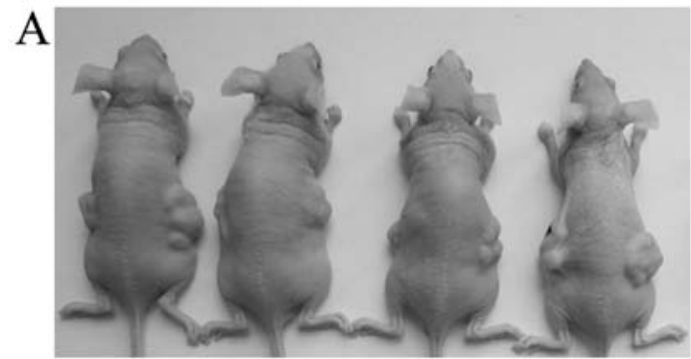

Left: Eps8 shRNA; Right: NC shRNA

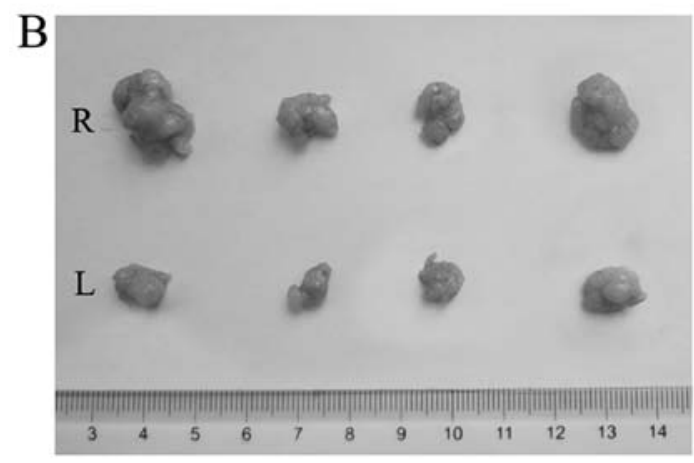

$\mathrm{C}$

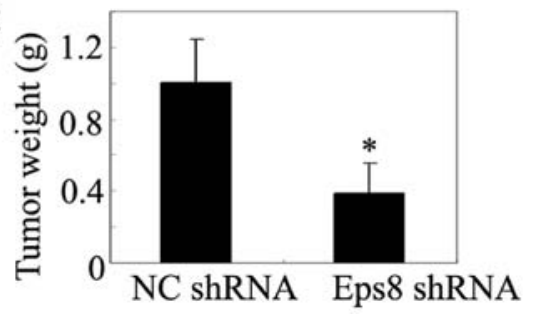

Figure 4. Eps8 knockdown results in decreased cell proliferation of MDAMB-231 cells in vivo. (A and B) Representative images of nude mice and the tumors following injection of $\sim 10^{7}$ of NC shRNA-infected cells (right dorsal flank) and Eps8 shRNA1-infected cells (left dorsal flank). (C) The measurement of volume of the excised tumors. These data are mean \pm SD in 3 independent experiments. " $\mathrm{p}<0.05$ compared with controls.

tion of S-phase cells were even lower in Eps8 repressed cells compared with NC control cells (44.83\%) (Fig. 3F). These observations suggested that Eps8 knockdown arrested cells at the G1/S checkpoint. Therefore, the above data showed that Eps8 knockdown suppresses proliferation, and cell cycle progression enhancing cell apoptosis of MDA-MB-231 cells.

The inhibitory effect of Eps8 knockdown on breast cancer cell proliferation in vitro suggested that Eps8 deletion might suppress tumor growth in vivo. Tumorigenicity assays were performed by subcutaneous injection of Eps8 shRNA cells into nude mice, and NC shRNA cells were used as controls. Within 4 weeks, solid tumors were readily visible in left and right dorsal regions of all mice (Fig. 4A), but the average tumor volume and weight of the Eps8 shRNA group were markedly reduced by $>60 \%$ compared with negative controls (Fig. $4 \mathrm{~B}$ and $\mathrm{C}$ ). Thus, the above data showed Eps8 knockdown could decrease breast cancer cell proliferation in vivo.

Eps8 knockdown suppresses breast cancer cell migration, decreases the number and size of EGF-induced filopodia and increases the sensitivity of breast cancer cells to cisplatin. We next assessed the effect of Eps8 on breast cancer cell migration by wound-healing assays. As shown in Fig. 5A, Eps8 depletion produced $62 \%$ inhibition of cell migration in MDA-MB-231 cells. In contrast, control groups dramatically promoted cell migration. Thus, Eps8 knockdown was able to significantly inhibit cell motility, which confirmed the role of Eps8 in breast cancer cell migration.

As Eps8 was previously described to be essential for actin dynamics and cytoskeletal organization in pancreatic cancer (12), we examined the effects of Eps8 knockdown on filopodia in MDA-MB-231 cells. Cells were cultivated for $48 \mathrm{~h}$ in serum-free medium and then EGF was added for $30 \mathrm{~min}$. Eps8-deletion cells exhibited reduction of filopodial density and length and inhibited the filopodial growth (Fig. 5B), indicating that Eps8 is involved in a rearrangement of F-actin in breast cancer cells.

We further investigated the implication of Eps8 in chemosensitivity of breast cancer cells. MDA-MB-231 cells were treated with cisplatin for $24 \mathrm{~h}$. Sixty-two percent reduction of MTT absorbance was detected in Eps8 shRNA cells compared with $48 \%$ inhibition in their control cells (Fig. 5C). Thus, Eps8 knockdown was able to sensitize MDA-MB-231 cells to the cytotoxicity of cisplatin as reported in cervical cancer cells (8).

Eps8 affects the expression of ERK signaling, MMP9, p53 and EMT markers. Because Eps8 influences many genes involved in the development and progression of human cancers, we decided to examine whether Eps8 regulates these target genes in breast cancer cells. As shown in Fig. 6A, Eps8 knockdown had no effect on ERK protein, but it decreased the phosphorylated level of ERK and downregulated the expression of c-Myc and cyclin D1 (CCND1) as downstream mitogenic targets of ERK signaling (26). Moreover, Eps8 knockdown exhibited inhibited MMP9 and enhanced the tumor suppressor p53. The level of EMT epithelial marker E-cadherin was concomitantly increased in Eps8-depleted cells. In contrast, Eps8 knockdown decreased the levels of mesenchymal markers N-cadherin and vimentin. Taken together, these data showed that Eps8 regulates breast cancer cell proliferation and migration, at least in part, by affecting ERK signaling, MMP9, p53 and EMT markers.

\section{Discussion}

Eps 8 is important in regulating the development and progression of many human cancers. However, the role of Eps8 in human breast cancer has not been reported. In the present study, we examined the expression level of Eps8 in histological samples from a large number of patients with previously untreated breast cancer. As detected in the immunohistochemistry analysis, Eps8 was overexpressed in most of breast cancer tissues. Moreover, Eps 8 protein was significantly higher in $55.6 \%$ of stages II to III breast cancer samples compared to normal mammary tissues. Furthermore, 5 breast cancer cell lines were detected to confirm that the protein level of Eps8 is high in highly invasive breast cancer cells, and low or lost in weakly invasive breast cancer cells. Thus, Eps8 expression may predict cancer recurrence and outcome in stages II-III breast cancer patients.

Evidence presented here demonstrated the effect of Eps8 on breast cancer cell proliferation, we overexpressed 


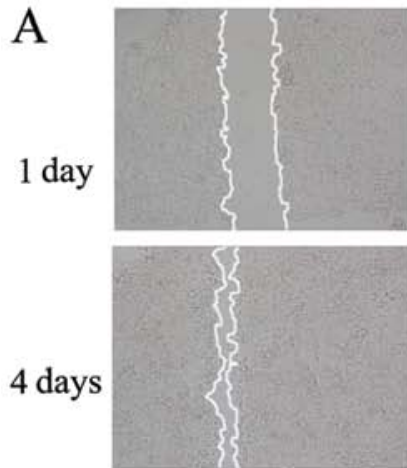

Mock
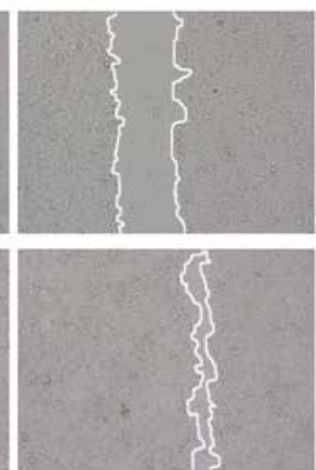

NC shRNA-LV
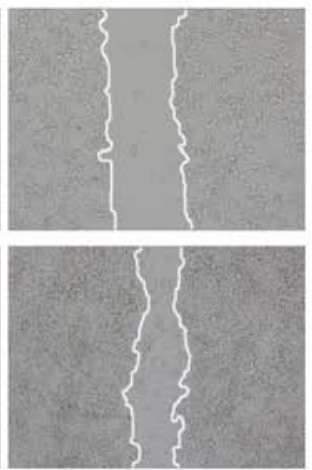

Eps8 shRNA-LV
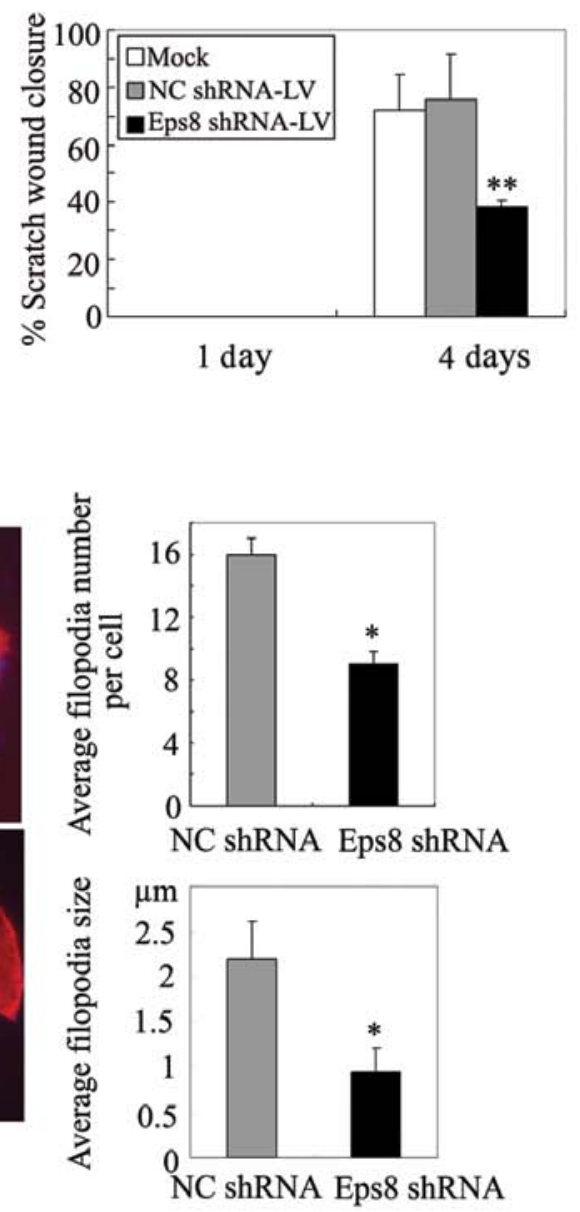

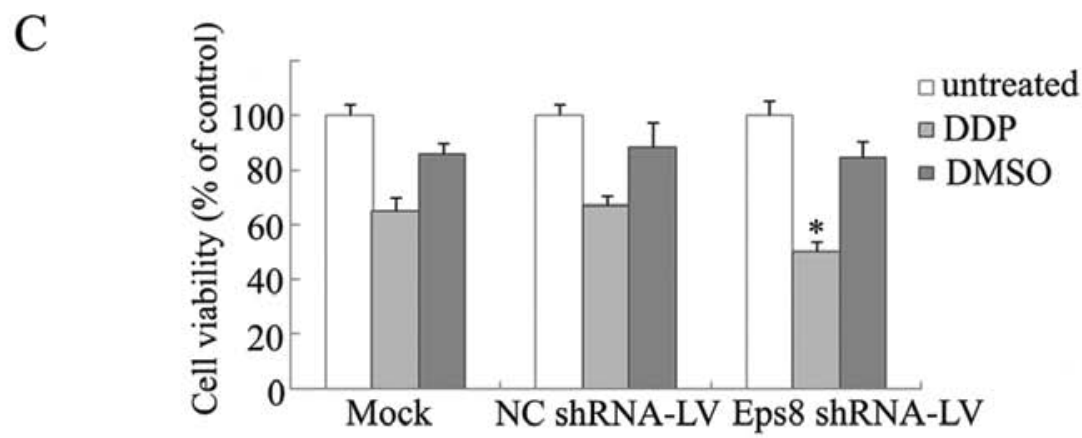

Figure 5. Eps8 regulates migration and the sensitivity to cisplatin of MDA-MB-231 cells. (A) Representative images of wound-healing assays were obtained at the indicated time following initiation of the scratch in lentivirus-infected and parental MDA-MB-231 cells. These areas were calculated by AutoCAD software. (B) Representative images of subcellular localization of actin structures in lentivirus-infected and parental MDA-MB-231 cells. Cells were serum deprived for $48 \mathrm{~h}$ and stimulated with $60 \mathrm{ng} / \mathrm{ml}$ of EGF for $30 \mathrm{~min}$ to induce filopodia. F-actin was stained by Alexa fluor 595 conjugated to phalloidin whereas Hoechst 33258 was used to stain the nucleus. Mean number and size of the filopodia were from at least 20 cells. (C) MTT assays in lentivirus-infected and parental MDA-MB-231 cells treated with $30 \mu \mathrm{M}$ of cisplatin and control agent DMSO. Twenty-four hours later, cell viability was measured. ${ }^{*}<0.05$, compared with parental and control cells.

Eps8/GFP in MCF7 cell line with low expression of Eps8 to investigate the role of Eps8 in breast cancer cellular growth in vitro. Eps8 expression significantly promoted cell proliferation. In contrast, we used lentivirus-based RNAi system to knock down Eps8 expression in MDA-MB-231 cell line with high expression of Eps8. Eps8 attenuation reversed the growth phenotype of Eps8-overexpressing breast cancer cells and increased the sensitivity of breast cancer cells to cisplatin. Our data indicated that Eps8 expression is critical for cell growth and proliferation of breast cancer cells in vitro and in vivo and possibly involved in the development and progression in human breast cancer.

Actin polymerization and formation of lamellipodia are believed to play important roles in cell migration during metastasis (27). Eps8, Abi-1 and Sos1 form a tricomplex, induce Rac-specific guanine nucleotide exchange factor (GEF) 


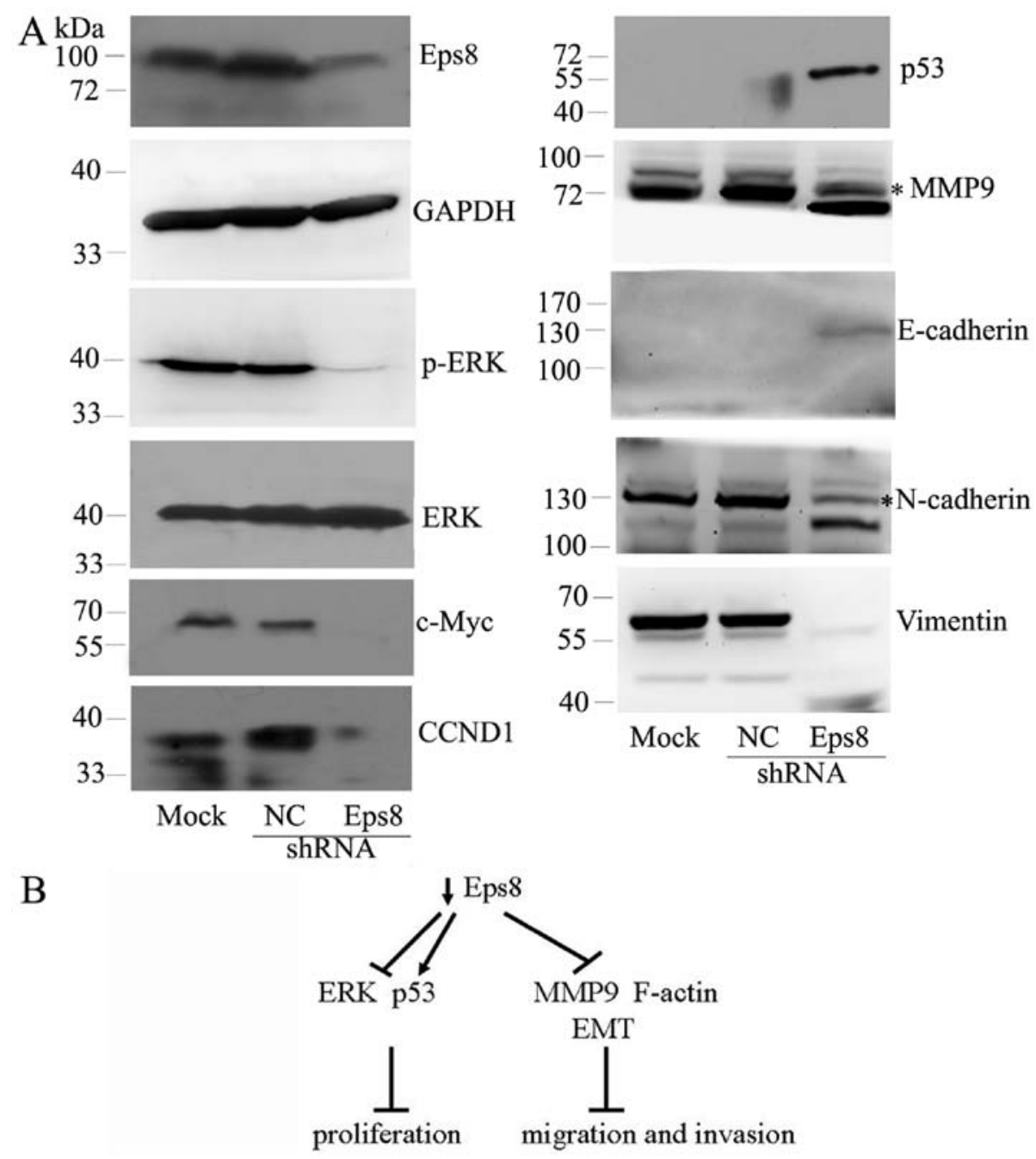

Figure 6. Eps8 modulates the expression of multiple downstream target genes. (A) Comparison of the expression of phospho ERK, total ERK, p53, MMP9 and EMT markers in lentivirus-infected and parental MDA-MB-231 cells was performed by Western blotting. GAPDH was used as a loading control for total lysate samples. (B) Proposed model illustrating the oncogenic effect of Eps8 participating in the progression of breast cancer. Eps8 knockdown decreases the expression of active ERK and MMP9 but increases the activity of p53 required for the proliferation and migration. Eps 8 knockdown promotes the expression of epithelial protein E-cadherin, whereas inhibits the expression and activity of mesenchymal proteins including N-cadherin and vimentin required for cell invasion.

activity and transduce signals from Ras to Rac leading to actin remodeling (28-30). Moreover, Eps8 binds with F-actin and colocalizes with actin-based membrane protrusions such as lamellipodia, filopodia and membrane ruffles $(28,29)$. Likely, Eps8 knockdown reduces filopodial density and length and inhibits cell migration in breast cancer cells, suggesting the important role of Eps8 in cellular movement and migration of tumor cells.

Eps8 is an important signal molecule and integrates multiple pathways. For example, Eps8 could control the Ras-Raf-MEK-ERK signaling cascade (31), which plays a crucial role in regulating cellular processes including differentiation, proliferation, survival and apoptosis (32). We demonstrated that Eps8 affects the expression of phosphorylated ERK and downstream genes c-Myc and CCND1 in cellular growth of breast cancer. Apart from regulating cell survival, the ERK pathway promoted invasiveness in tumor cells by upregulation of MMPs such as MMP9 for extracellular matrix remodeling (33). We provided evidence that the levels of MMP9 are decreased in Eps8 shRNA infected cells. In addition, the tumor suppressor gene p53 inhibits the development and growth of the majority of human tumors (34). Eps8 knockdown also enhanced p53 upregulation, resulting in growth arrest or apoptosis of breast cancer cells. The results supported the conclusion that Eps8 regulates the expression of c-Myc, CCND1 and MMP9 through the ERK pathway as well as p53.

Cancer progression toward malignancy is mostly associated with the loss of epithelial differentiation and a switch toward a mesenchymal phenotype. Epithelial-mesenchymal transition (EMT) is considered a critical process for tumor invasion and metastasis (35). Many epithelial proteins including E-cadherin, $\alpha$-catenin and $\beta$-catenin were downregulated, whereas mesenchymal proteins were upregulated, such as fibonectin, $\mathrm{N}$-cadherin, $\alpha$-SMA and vimentin (36). Loss of E-cadherin promotes metastasis by disrupting intercellular contacts in metastatic dissemination (37). N-cadherin promotes proliferation, adhesion and invasion of prostate cancer cells (38). Moreover, vimentin is associated with a highly invasive cellular phenotype (39). In the present study 
we found that Eps8 knockdown has a significant impact on EMT by increasing E-cadherin expression and decreasing $\mathrm{N}$-cadherin and vimentin, and might achieve lower motility and invasiveness (Fig. 6B). Therefore, these data suggested that Eps8 might partly mediate EMT-like transition and invasion in breast cancer.

In conclusion, our results revealed the pivotal role of Eps8 in breast cancer progression. Eps8 was highly expressed in breast cancer tissues and highly-invasive cell lines. Eps8 regulated the proliferation, migration and invasion of breast cancer cells in vitro and in vivo. Eps8 knockdown inhibited cell migration, decreased the number and size of EGF-induced filopodin and increased the cytotoxicity of cisplatin in breast cancer cells, at least in part, by affecting the expression of ERK signaling, MMP9, p53 and EMT markers. The oncoprotein Eps8 might have an important role as a molecular therapeutic target and even prognostic marker in human breast cancer.

\section{Acknowledgements}

This study was supported by the National Natural Science Foundation of China (nos. 81272318 and 81272190), and the Scientific Research Fund of Hunan Provincial Education Department (no. 13B068).

\section{References}

1. Bray F, McCarron P and Parkin DM: The changing global patterns of female breast cancer incidence and mortality. Breast Cancer Res 6: 229-239, 2004.

2. Gupta GP and Massague J: Cancer metastasis: building a framework. Cell 127: 679-695, 2006.

3. Dahiya R and Deng G: Molecular prognostic markers in breast cancer. Breast Cancer Res Treat 52: 185-200, 1998.

4. Ross JS and Fletcher JA: The HER-2/neu oncogene in breast cancer: prognostic factor, predictive factor, and target for therapy. Oncologist 3: 237-252, 1998.

5. Sgouros J, Galani E, Gonos E, et al: Correlation of nm23-H1 gene expression with clinical outcome in patients with advanced breast cancer. In Vivo 21: 519-522, 2007.

6. Barnes DM and Gillett CE: Cyclin D1 in breast cancer. Breast Cancer Res Treat 52: 1-15, 1998.

7. Wang H, Teh MT, Ji Y, et al: EPS8 upregulates FOXM1 expression, enhancing cell growth and motility. Carcinogenesis 31: 1132-1141, 2010.

8. Chen YJ, Shen MR, Chen YJ, Maa MC and Leu TH: Eps8 decreases chemosensitivity and affects survival of cervical cancer patients. Mol Cancer Ther 7: 1376-1385, 2008.

9. Maa MC, Lee JC, Chen YJ, et al: Eps8 facilitates cellular growth and motility of colon cancer cells by increasing the expression and activity of focal adhesion kinase. J Biol Chem 282 19399-19409, 2007.

10. Wang H, Patel V, Miyazaki H, Gutkind JS and Yeudall WA: Role for EPS8 in squamous carcinogenesis. Carcinogenesis 30 $165-174,2009$.

11. Zhang W, Wang L, Liu Y, et al: Structure of human lanthionine synthetase C-like protein 1 and its interaction with Eps8 and glutathione. Genes Dev 23: 1387-1392, 2009.

12. Welsch T, Endlich K, Giese T, Buchler MW and Schmidt J: Eps8 is increased in pancreatic cancer and required for dynamic actinbased cell protrusions and intercellular cytoskeletal organization. Cancer Lett 255: 205-218, 2007.

13. Ding X, Zhou F, Wang F, et al: Eps8 promotes cellular growth of human malignant gliomas. Oncol Rep 29: 697-703, 2013.

14. Kang H, Wilson CS, Harvey RC, et al: Gene expression profiles predictive of outcome and age in infant acute lymphoblastic leukemia: a Children's Oncology Group study. Blood 119: 1872-1881, 2012.

15. Matoskova B, Wong WT, Salcini AE, Pelicci PG and Di Fiore PP: Constitutive phosphorylation of eps 8 in tumor cell lines: relevance to malignant transformation. Mol Cell Biol 15: 3805-3812, 1995.
16. Chu PY, Liou JH, Lin YM, et al: Expression of Eps8 correlates with poor survival in oral squamous cell carcinoma. Asia Pac J Clin Oncol 8: e77-e81, 2012.

17. Yang TP, Chiou HL, Maa MC and Wang CJ: Mithramycin inhibits human epithelial carcinoma cell proliferation and migration involving downregulation of Eps8 expression. Chem Biol Interact 183: 181-186, 2010.

18. Gorsic LK, Stark AL, Wheeler HE, et al: EPS8 inhibition increases cisplatin sensitivity in lung cancer cells. PLoS One 8: e82220, 2013.

19. Abdel-Rahman WM, Ruosaari S, Knuutila S and Peltomaki P: Differential roles of EPS8 in carcinogenesis: loss of protein expression in a subset of colorectal carcinoma and adenoma. World J Gastroenterol 18: 3896-3903, 2012.

20. Li YH, Xue TY, He YZ and Du JW: Novel oncoprotein EPS8: a new target for anticancer therapy. Future Oncol 9: 1587-1594, 2013.

21. Yao J, Weremowicz S, Feng B, et al: Combined cDNA array comparative genomic hybridization and serial analysis of gene expression analysis of breast tumor progression. Cancer Res 66: 4065-4078, 2006.

22. Mangravite LM, Lipschutz JH, Mostov KE and Giacomini KM: Localization of GFP-tagged concentrative nucleoside transporters in a renal polarized epithelial cell line. Am J Physiol Renal Physiol 280: F879-F885, 2001.

23. Schmid JA, Birbach A, Hofer-Warbinek R, et al: Dynamics of NF kappa B and Ikappa Balpha studied with green fluorescent protein (GFP) fusion proteins. Investigation of GFP-p65 binding to $\mathrm{DNa}$ by fluorescence resonance energy transfer. J Biol Chem 275: 17035-17042, 2000

24. Lois C, Hong EJ, Pease S, Brown EJ and Baltimore D: Germline transmission and tissue-specific expression of transgenes delivered by lentiviral vectors. Science 295: 868-872, 2002.

25. Ding X, Yang Z, Zhou F, et al: Transcription factor AP-2alpha regulates acute myeloid leukemia cell proliferation by influencing Hoxa gene expression. Int J Biochem Cell Biol 45: 1647-1656, 2013.

26. Pan CC, Bloodworth JC, Mythreye K and Lee NY: Endoglin inhibits ERK-induced c-Myc and cyclin D1 expression to impede endothelial cell proliferation. Biochem Biophys Res Commun 424: 620-623, 2012.

27. Ridley AJ: Rho GTPases and cell migration. J Cell Sci 114: 2713-2722, 2001.

28. Scita G, Tenca P, Areces LB, et al: An effector region in Eps8 is responsible for the activation of the Rac-specific GEF activity of Sos-1 and for the proper localization of the Rac-based actinpolymerizing machine. J Cell Biol 154: 1031-1044, 2001.

29. Scita G, Nordstrom J, Carbone R, et al: EPS8 and E3B1 transduce signals from Ras to Rac. Nature 401: 290-293, 1999.

30. Chen H, Wu X, Pan ZK and Huang S: Integrity of SOS1/EPS8/ ABI1 tri-complex determines ovarian cancer metastasis. Cancer Res 70: 9979-9990, 2010.

31. Hecquet C,Lefevre G, Valtink M, Engelmann K and Mascarelli F: Activation and role of MAP kinase-dependent pathways in retinal pigment epithelial cells: ERK and RPE cell proliferation. Invest Ophthalmol Vis Sci 43: 3091-3098, 2002.

32. Chang F, Steelman LS, Lee JT, et al: Signal transduction mediated by the Ras/Raf/MEK/ERK pathway from cytokine receptors to transcription factors: potential targeting for therapeutic intervention. Leukemia 17: 1263-1293, 2003.

33. McCawley LJ, Li S, Wattenberg EV and Hudson LG: Sustained activation of the mitogen-activated protein kinase pathway. A mechanism underlying receptor tyrosine kinase specificity for matrix metalloproteinase-9 induction and cell migration. J Biol Chem 274: 4347-4353, 1999.

34. Oren M: Decision making by p53: life, death and cancer. Cell Death Differ 10: 431-442, 2003.

35. Thiery JP: Epithelial-mesenchymal transitions in development and pathologies. Curr Opin Cell Biol 15: 740-746, 2003.

36. Grunert $S$, Jechlinger $M$ and Beug H: Diverse cellular and molecular mechanisms contribute to epithelial plasticity and metastasis. Nat Rev Mol Cell Biol 4: 657-665, 2003.

37. Onder TT, Gupta PB, Mani SA, et al: Loss of E-cadherin promotes metastasis via multiple downstream transcriptional pathways. Cancer Res 68: 3645-3654, 2008.

38. Tanaka H, Kono E, Tran CP, et al: Monoclonal antibody targeting of $\mathrm{N}$-cadherin inhibits prostate cancer growth, metastasis and castration resistance. Nat Med 16: 1414-1420, 2010.

39. Lang SH, Hyde C, Reid IN, et al: Enhanced expression of vimentin in motile prostate cell lines and in poorly differentiated and metastatic prostate carcinoma. Prostate 52: 253-263, 2002. 